\title{
Utilization of FIB Technique in TEM Specimen Preparation of GaN-based Devices for Dislocation Investigation
}

\author{
Jian-Guo Zheng ${ }^{1}$, Zhenguang Shao ${ }^{2}$ and Dunjun Chen $^{2}$ \\ ${ }^{1}$ Irvine Materials Research Institute, University of California, Irvine, CA 92697-2800, USA \\ ${ }^{2}$ Jiangsu Provincial Key Laboratory of Advanced Photonic and Electronic Materials, and School of \\ Electronic Science and Engineering, Nanjing University, Nanjing 210093, P.R. China
}

Dislocations can be frequently observed in GaN-based devices which have various applications in optoelectronics as well as high-power and high-frequency equipment. The properties of GaN-based devices can be affected by dislocations [1-4]. For example, thread dislocations may serve as a nonradiative recombination center and affect electron mobility. Different doped GaN layers in the devices are normally fabricated on a substrate, and dislocations (type and density) change in these layers. Therefore, it is necessary to study dislocations in GaN-based devices, especially in the active doped GaN layers, in order to understand device behavior and improve device performance.

Transmission electron microscopy (TEM) is almost an exclusive technique to observe high density dislocations inside the devices. TEM specimen preparation is the first crucial step toward a successful TEM experiment. To correlate device performance and dislocation information, a TEM specimen has to come from a specifically selected device (Fig. 1) and to be cut in some specific directions. Obviously, focus ion beam (FIB) technique available in a dual-beam system has a great advantage over traditional TEM specimen preparation techniques.

There still could be a lot of challenges to prepare a TEM specimen from a selected device by using FIB. Besides specific location, the TEM specimen needs to have a large thin area including all layers of devices ranging from the substrate to the top layer. For dislocation study, the TEM specimen also needs to be free of specimen bending in the large area of interest and be thinned down to electron transparency with similar thickness in the area. We have successfully applied FIB technique to prepare TEM specimens for dislocation study of GaN-based devices. Fig. 2 shows such an example, where the arrow indicates the interface between the GaN layers and sapphire substrate. The image intensity above the interface is quite uniform, indicating the GaN layers have similar thickness in the electron beam direction. Fig. 3 is a corresponding weak-beam TEM image showing clearly how threading dislocations vary from the interface to the top of the device. This presentation will summarize a few methods to prepare good TEM specimens for dislocation study by using FIB and to prevent some possible problems such as specimen bending (Fig. 4).

[1] S. Nakamura, Science 281 (1998), p. 956-961

[2] S. J. Rosner et al, Applied Physics Letters 70 (1997), 420

[3] N. G. Weimann and L. F. Eastman, J. Appl. Phys. 83(1998), 3656

[4] D. P. Han et al, Jpn. J. Appl. Phys. 54 (2015), 02BA01

[5] TEM work was performed at the Irvine Materials Research Institute (IMRI) at UC Irvine, using FEI Quanta 3D dual-beam system for TEM specimen preparation which was funded in part by the National Science Foundation Center for Chemistry at the Space-Time Limit under grant no. CHE0802913 


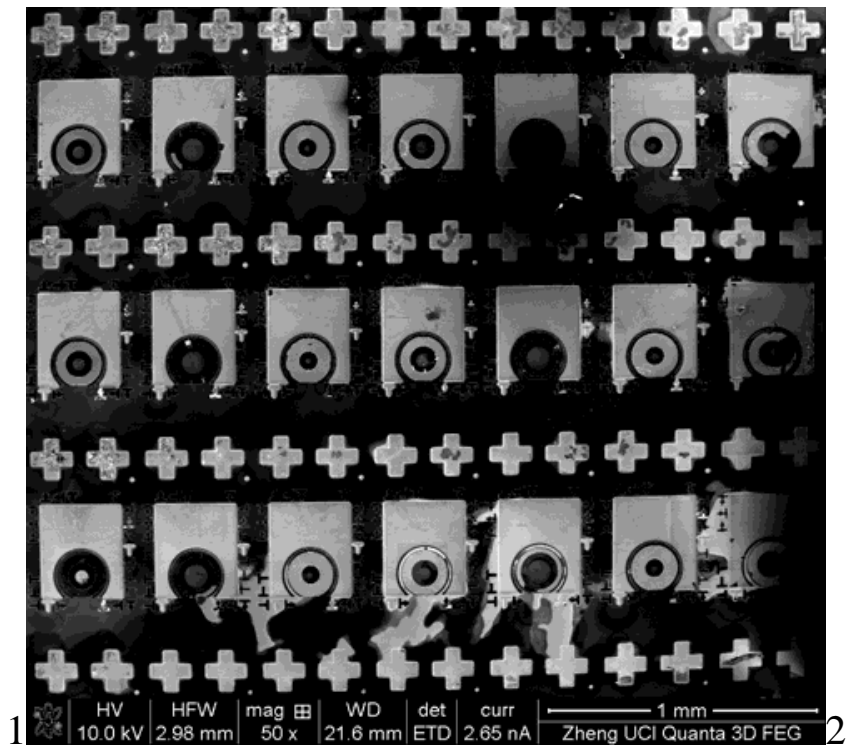

Fig. 1 An array of 7x3 GaN-based devices imaged by SEM.

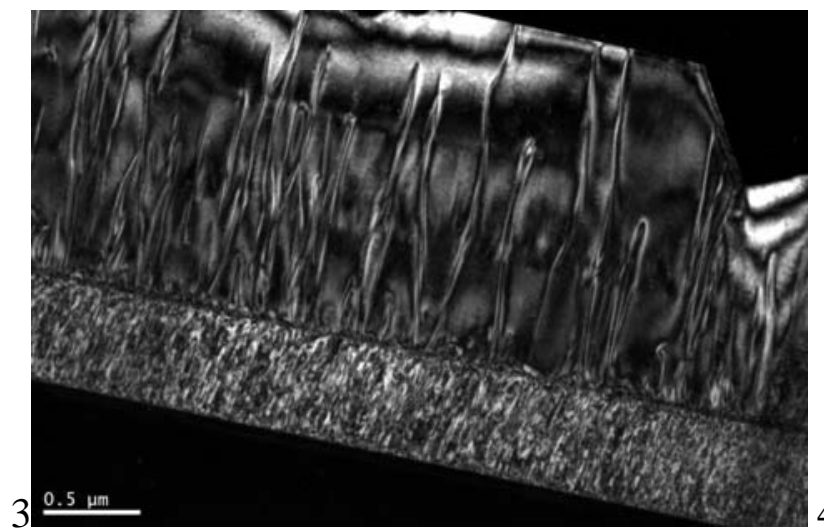

Fig. 3 Cross-sectional weak-beam TEM image of a GaN-based device shown in Fig. 2. Threading dislocations may pass through different doped GaN layers or terminate at the layer boundaries.

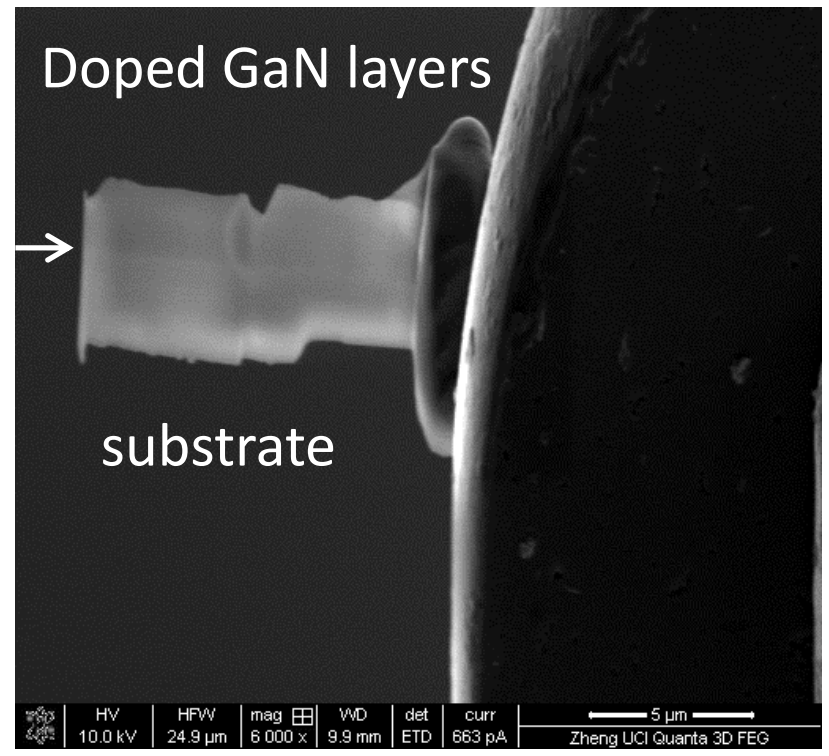

Fig. 2 SEM image of a cross-sectional TEM specimen of one GaN-based device prepared by using FIB. The arrow indicates the interface between sapphire substrate and doped GaN layers.

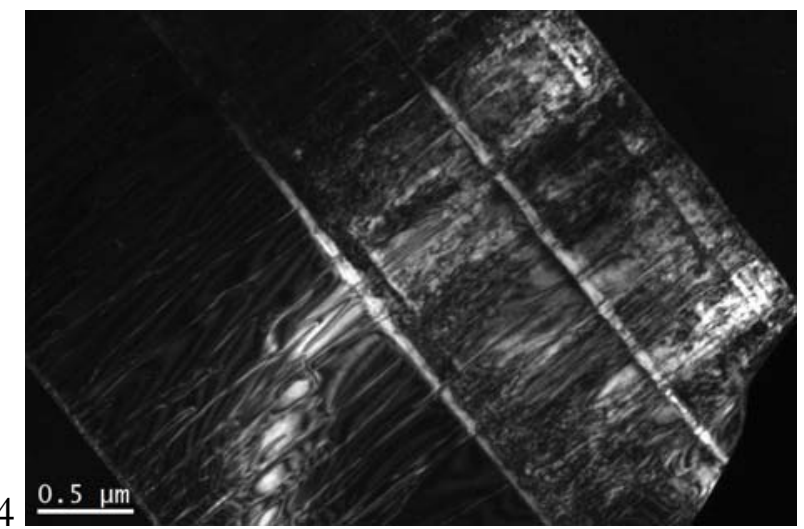

Fig. 4 Cross-sectional weak-beam TEM image of a GaN-based device, where the bend contour is visible as a broad bright band, indicating the specimen is bent. 